\title{
Quality of life and psychological assessment in patients with Fibromyalgia Syndrome during COVID-19 pandemic in Italy: prospective observational study
}

\author{
Vittorio Schweiger ${ }^{1}$, Erica Secchettin ${ }^{1, \star}$, Giovanni Perini ${ }^{1}$, Alvise Martini ${ }^{1}$, \\ Katia Donadello ${ }^{1}$, Leonardo Gottin ${ }^{1}$, Giovanna Del Balzo ${ }^{2}$, Giustino Varrassi ${ }^{3}$, \\ Enrico Polati ${ }^{1}$
}

${ }^{1}$ Department of Surgery, Dentistry,

Paediatrics and Gynaecology, University of Verona, 37134 Verona, Italy

${ }^{2}$ Department of Medicine and Public Health, University of Verona, 37134

Verona, Italy

${ }^{3}$ Fondazione Paolo Procacci and

European League against Pain, 00185

Rome, Italy

\section{*Correspondence}

erica.secchettin@univr.it

(Erica Secchettin)

\begin{abstract}
Introduction: Chronic pain patients who undergo to stressful events may experience worsening in pain, sleep, and quality of life (QoL). The primary objective of this observational study was to compare QoL and sleep parameters before and after the COVID-19 lockdown in patients with Fibromyalgia Syndrome (FMS). The psychological impact of lockdown was also assessed, as well as the emotional impact of the pandemic and its correlations with patient socio-demographics.

Methods: Patients aged $\geq 18$ years with FMS diagnosed according to ACR (American College of Rheumatology) 2016 Criteria with at least one pre-pandemic QoL and sleep evaluation were included. QoL and sleep disturbances were analyzed by comparing scores on the Fibromyalgia Impact Questionnaire-Revised (FIQ-R), the 12-item Short Form Survey (SF-12), and the Pittsburgh Sleep Quality Index (PSQI) before and after the first lockdown in Italy (March to May 2020). Psychological impact was investigated via a 52-item survey of daily life changes in FMS management during the lockdown and emotional impact with the Impact Event Scale-Revised (IES-R) tool during the onset period after the lockdown. Questionnaire responses were correlated with patients' sociodemographics.

Results: Questionnaires were submitted via email to 54 patients; $37 / 54$ patients (63.7\%) returned them. QoL and sleep disturbances showed no statistically significant worsening. However, the psychological impact survey revealed that 3 out of 5 patients feared very much for their family members' lives during lockdown. The emotional impact survey disclosed $72.7 \%$ of patients with psychological distress.

Conclusions: While questionnaire responses showed no significant changes in QoL and sleep after the COVID-19 lockdown in this sample of FMS patients, the emotional investigation revealed moderate/severe psychological distress not detected by commonly used QoL tests in FMS.
\end{abstract}

\section{Keywords}

Fibromyalgia; Quality of life; Depression; Sleep; COVID-19 pandemic

\section{Introduction}

The fibromyalgia syndrome (FMS) is a chronic disease affecting an estimated $2-4 \%$ of the general population. Clinical features are comprised within a broad spectrum of symptoms, including widespread pain, fatigue, disturbed or unrefreshing sleep, gut and urological disorders, mood changes, muscle stiffness, and cognitive deficits [1]. FMS negatively impacts on quality of life $(\mathrm{QoL})$ by reducing work skills and increasing both health and welfare costs $[2,3]$. No single triggering cause for FMS has been identified; it is thought to have a multifactorial etiology together with predisposing and precipitating factors, such as stressors related to the onset or worsening of
FMS, both physical (trauma, motor vehicle accidents, surgery, sexual abuse, violence, infections) and psychological (warfare, stalking, mobbing, job loss, family problems, psychological violence, bereavement, school failure) [4]. This hypothesis has been corroborated in experimental stress-induced murine models [5]. FMS patients who have survived catastrophic events or natural disasters may experience changes in pain, sleep, fatigue, and QoL [6, 7]. In most parts of the world with cases of COVID-19, measures to reduce transmission of the virus have altered usual activities, routines, and livelihoods, and have had a significant impact on mental health and quality of life [8-12]. The aim of this prospective, longitudinal observational study, was to determine whether the COVID-19 pandemic and related 
life changes have influenced the health status of FMS patients referred to our Pain Therapy Centre, as expected considering the fragility of this population.

\section{Methods}

\subsection{Study model}

Prospective, longitudinal, observational, single centre study. The observation was conducted at the University Hospital of Verona, Department of Anaesthesia and Intensive Care, Pain Therapy Centre. Participants were informed about the purpose of the study; informed written consent was obtained before enrolment. The study was approved by the local Clinical Research Committee (COVIDFIBRO study, ID 2890CESC) and complied with the revised ethical guidelines of the Declaration of Helsinki.

\subsection{Study population}

Inclusion criteria were age $\geq 18$ years, with FMS diagnosed according to ACR (American College of Rheumatology) 2016 criteria (Widespread Pain Index WPI $\geq 7$ and Symptom Severity $\mathrm{SS} \geq 5$ ) or WPI $4-6$ and $\mathrm{SS} \geq 9$ in at least 4 out of 5 body areas, symptoms present for at least 3 months) with a QoL and sleep evaluation performed during the prepandemic period. Exclusion criteria were lack of at least one test (FIQ-R, SF12, PSQI) in the period from 01.01.2019 to 01.01.2020. At the end of the COVID-19 lockdown (May 2020), a computerized version of the Fibromyalgia Impact Questionnaire-Revised (FIQ-R) [13], the Short Form 12 (SF-12) [14], the Pittsburgh Sleep Quality Index (PSQI) [15], and the Impact Event ScaleRevised (IES-R) [16] was e-mailed to the study participants, together with a 52 -item survey (21 with yes/no responses, 31 with a 5-point response on a Likert-like scale) investigating changes in personal and family daily life during the lockdown. Socio-demographic data were collected at enrolment.

\subsection{Study objectives}

The primary study objective was to compare changes in QoL and sleep in FMS patients before and after the COVID-19 lockdown as measured with responses to the FIQ-R, SF-12, and PSQI. The secondary objective was to assess psychological and emotional status via the 52-item survey and the IES-R tool. Questionnaire responses were correlated with patients' sociodemographic data.

\subsection{Statistical analysis}

Statistical analysis was performed using R software (RStudio PBC) Version 1.3.959. The Shapiro-Wilk test was used to determine normal distribution of the sample. Data are presented as the median and interquartile range (IQR) or the mean \pm standard deviation (SD), as appropriate, or like frequencies. The Mann-Whitney- Wilcoxon test was used to compare paired data for QoL scores before and after lockdown. Fisher's exact test was used to test associations between two categorical variables. Pearson's correlation was used to evaluate the statistical relationship between QoL and IES-R. Survey responses are reported as descriptive analysis.

\section{Results}

\subsection{Study population}

Computerized questionnaires were e-mailed to 54 patients in this study sample, 37 (63.7\%) of which completed and returned the questionnaires and were included in the analysis. Table 1 presents the patients' demographics of these population, which is highly representative of our FMS population as reported in a previous epidemiological observation [17]. Most were married or cohabiting (59.5\%), with an educational level of high school diploma or above (78.4\%). The majority had a stable job before the pandemic $(70.3 \%)$, while less than $50 \%$ worked during the lockdown period. The mean duration of FMS was 8.5 years (SD 5.45) and disease severity was high or very high in most patients $(62.2 \%)$, with a mean FIQ-R score $>63$. One patient reported being positive to SARSCOV-2 infection and 5 patients (13.5\%) reported cold symptoms/dyspnea/anosmia/ageusia despite a COVID-19-negative swab test. Most patients $(n=24,64.8 \%)$ had to postpone medical examinations and requests for psychological/psychiatric consultation ( $\mathrm{n}=11,29.7 \%)$ due to fear of contracting an infection.

TA B L E 1. Patient demographics.

\begin{tabular}{|c|c|}
\hline Characteristic & $\mathrm{N}=37(\%)$ \\
\hline \multicolumn{2}{|l|}{ Sex } \\
\hline Female & 34 (91.9) \\
\hline Male & $3(8.1)$ \\
\hline \multirow[t]{2}{*}{$\mathrm{F}: \mathrm{M}$ ratio } & $11: 1$ \\
\hline & mean $\pm \mathrm{SD}$ \\
\hline Age, yr. & $50.3 \pm 11.65$ \\
\hline FIQ-R, 0-100 & $67.5 \pm 15.26$ \\
\hline PSQI, 0-21 & $12.63 \pm 4.24$ \\
\hline SF12 (PCS) & $27.49 \pm 7.27$ \\
\hline SF12 (MCS) & $40.54 \pm 10.65$ \\
\hline FM onset, yr. & $8.5 \pm 5.45$ \\
\hline \multicolumn{2}{|c|}{$\begin{array}{l}\text { FIQR, Fibromyalgia Impact Questionnaire } \\
\text { Revised; PSQI, Pittsburgh Sleep Quality } \\
\text { Index; SF12, 12-item Short-Form Health } \\
\text { Survey; MCS, Mental Component Sum- } \\
\text { mary; PCS, Physical Component Summary. }\end{array}$} \\
\hline
\end{tabular}

\subsection{Impact of COVID-19 pandemic on QoL}

There was no statistically significant difference in median FIQ$\mathrm{R}$ scores for QoL before and after lockdown (69.4, IQR 54.380.1 vs. 71.3 , IQR $59.8-79.3 ; p=0.3909)$. No associations were found between lower FIQ-R score $(\mathrm{n}=16,43.2 \%)$ and demographics, except for lower educational level $(p=0.0135)$. There was no statistically significant difference in the median SF-12 Physical Component Summary (PCS) score before and after lockdown (28.19, IQR 23.8-31.9 vs. 28.76, IQR 24.535.4; $p=0.4651)$. There was no statistically significant difference in the median SF-12 Mental Component Summary (MCS) score before and after lockdown (38.88, IQR 32.4-50.9 
vs. 35.55, IQR 27-44.6, $p=0.4651)$. There was no difference in the median PSQI score before and after lockdown (13.5, IQR $10.3-16$ vs. 13 , IQR $10-15 ; p=0.1114)$. Analysis of responses to the IES-R showed that 22/37 (59.5\%) patients experienced the pandemic as a stressful event (mean IES-R score 39.14 \pm 15.13 ) (Table 2); 16/22 (72.7\%) had an IES-R score $\geq 33$, which is the cut-off commonly correlated with a diagnosis of post- traumatic stress disorder (PTSD). A negative correlation was found between the SF-12 MCS score and the IES-R, for both the total score and on the intrusion, hyperarousal subscale, indicating that the worse the mental health, the greater the psychological distress due to stressful events, including the effects of the current pandemic $(p<0.05)$. The 52-item tool investigating psychological impact in the anxiety domain showed that 23 patients $(62.2 \%)$ feared very much for their family members' lives and 19 (51.4\%) worried about infecting family members during the pandemic.

Differently, in the QoL domain, 20 patients (54\%) appreciated the lockdown period because "life was less hectic" and 16 $(43.2 \%)$ because "it allowed them to take up new hobbies". In the pain domain, 15 patients $(40.5 \%)$ reported having increased their use or started using painkillers during the lockdown. Finally, $81.1 \%$ of patients reported deterioration of sleep quality and duration.

\section{Discussion}

The first COVID-19 lockdown, in Italy, started in March and ended in May 2020. It has been recently shown that the prolonged lockdown and the urgency to drastically reduce social contacts determined negative effects on emotional and behavioural wellbeing in the Italian population, in particular for people with specific psychological characteristics such as lower emotional stability or higher alexithymia [18, 19]. According to many studies, FMS patients share common personality traits such as alexithymia and the tendency to experience negative feelings [20] and were thus expected to suffer from a particular psychological distress, due to the 2020 social distancing measures. Our preliminary data show no substantial differences in QoL and sleep quality as measured with three commonly used tools (FIQ-R, PSQI, SF-12) in FMS patients before and after the COVID-19 lockdown. This observation is shared by the findings from a recent study [21] but not by other investigations which reported that natural disasters had a negative impact on the QoL of FMS patients who survived the event, while these studies compared FMS patients exposed and not exposed to earthquake disaster [6] and FMS patients versus healthy patients and patients with other rheumatological diseases [7]. Similar conflicting results were noted in other patient's population. For instance, in cancer patients, pandemic outbreak showed no QoL worsening in some studies, while in other observations COVID-19 lockdown negatively affected QoL in terms of fear of tumor progression, risk of SARS CoV2 infection, financial difficulties or chemotherapy discontinuation $[22,23]$. Conversely, on closer analysis of the responses to the IES-R and the 52-item survey investigating the changes in perception of well-being at follow-up visits after lockdown, we found that the pandemic had a psychological and an emotional impact on most of the FMS patients. The IES-
$\mathrm{R}$ scores suggested moderate-severe psychological distress in many patients, while the survey revealed that the pandemic impacted on QoL in other areas (anxiety, pain, sleep) not detected by commonly used FMS questionnaires. Only the SF-12 MCS scores suggested a correlation with the total IES$\mathrm{R}$ score, underlying the psychological distress caused by the pandemic in these FMS patients. Assessment of the pandemic's psychological impact during and after lockdown in the general population showed a lower percentage of IES-R scores above the cut-off $(\geq 33)$ compared to the scores recorded for our FMS sample; however, these data are not conclusive due to the difference in sample size [24, 25]. Nevertheless, although commonly used in studies investigating the psychological impact of the COVID-19 pandemic, IES-R scores may be correlated to a diagnosis of PTSD, with a cut-off of 33 only for events "that involved death or threatened death" according to the Diagnostic and Statistical Manual of Mental Disorders (DSM-5) [26]. Job loss due to the pandemic and reduced social intercourse during lockdown, for example, do not meet PTSD diagnostic criteria. In such cases, the IES-R can be useful to explore the presence of an adjustment disorder, where patients experience more stress than would normally be expected in response to a stressful or unexpected event, and the stress causes significant problems in relationships, work or school [27].

There are several reasons for the discrepancy between standardized QoL and sleep quality tests and the psychological burden we found in our FMS population. Standardized tests investigating the QoL of FMS patients might not adequately explore the psychological and the emotional component that require additional assessment tools. The FIQ-R is primarily focused on the functional impact of the disease, while the SF-12, a general shorter form of the original SF-36 health questionnaire, should be supplemented with more specific QoL tools in vulnerable populations [28, 29]. Finally, the PSQI may not cover all aspects of sleep quality, especially the number of awakenings, which is one of the main causes of sleep disturbance in FMS [30]. Furthermore, the discrepancy might result from a kind of "engaging" communication with the physician, which is more complex to measure. Some observers have suggested that pain catastrophizing may be a communicative function that chronic pain patients use to express distress about their pain which they want to have recognized and managed within their social and medical context [31]. This particular concern deserves further investigation, for example, by means of computerized analysis of speech transcripts and their correlation with FMS QoL validated scales.

\subsection{Study strengths}

A strong point of the present study that distinguishes it from previous works is that participants could express via their IES$\mathrm{R}$ responses which aspect(s) of the pandemic they experienced as traumatic (i.e., fear of their own death or that of a family member) or as stressful (i.e., job loss or loneliness during lockdown). 
TA B L E 2. IES-R results.

\begin{tabular}{lcccccccc} 
Variable & \multicolumn{2}{c}{ Total score } & \multicolumn{2}{c}{ Intrusion } & \multicolumn{2}{c}{ Avoidance } & \multicolumn{2}{c}{ Hyperarousal } \\
Stressor & mean $(\mathrm{SD})$ & median $(\mathrm{IQR})$ & mean (SD) & median (IQR) & mean (SD) & median (IQR) & mean (SD) & median $(\mathrm{IQR})$ \\
Total $(\mathrm{n}=37)$ & $40.76(15.69)$ & $43(32-52)$ & $2.07(0.96)$ & $2.13(1.38-2.75)$ & $1.55(0.73)$ & $1.5(1.13-2)$ & $2.02(0.84)$ & $2(1.33-2.83)$ \\
\hline Pandemic-related $(\mathrm{n}=22)$ & $39.14(15.13)$ & $41(29.75-52.25)$ & $2.00(0.9)$ & $1.94(1.35-2.69)$ & $1.39(0.71)$ & $1.38(0.85-1.82)$ & $2.11(0.84)$ & $2(1.46-2.87)$ \\
\hline Financial loss $(\mathrm{n}=8)$ & $40.5(9.86)$ & $40(32.3-46.8)$ & $2.02(0.7)$ & $1.82(1.47-2.32)$ & $1.45(0.28)$ & $1.44(1.22-1.57)$ & $2.14(0.75)$ & $1.92(1.58-2.87)$ \\
\hline Afraid of infection $(\mathrm{n}=5)$ & $33(14.71)$ & $33(22-33)$ & $1.63(1)$ & $1.25(1.13-1.5)$ & $1.08(0.38)$ & $1.13(0.75-1.38)$ & $1.9(0.74)$ & $2(1.5-2)$ \\
Social isolation $(\mathrm{n}=7)$ & $43.14(19.99)$ & $49(41-55.5)$ & $2.32(1.11)$ & $2.5(2.19-2.75)$ & $1.45(1.02)$ & $1.75(0.69-2.06)$ & $2.3(1.08)$ & $2.6(2.25-2.92)$ \\
\hline Activities suspended $(\mathrm{n}=2)$ & $35(22.63)$ & $35(27-43)$ & $1.69(1.15)$ & $1.69(1.29-2.1)$ & $1.76(1.59)$ & $1.75(1.19-2.32)$ & $1.84(0.94)$ & $1.84(1.5-2.17)$ \\
Non-pandemic-related $(\mathrm{n}=15)$ & $43.13(16.72)$ & $43(32.5-52.5)$ & $2.16(1.03)$ & $2.25(1.56-2.49)$ & $1.8(0.73)$ & $1.88(1.56-2.12)$ & $1.91(0.88)$ & $1.60(1.33-2.5)$ \\
\hline
\end{tabular}

$S D$, mean \pm standard deviation. IQR, interquartile range (IQR). 


\subsection{Study limitations}

Limitations of the study were the low return rate, probably related to the use of computerized questionnaires, and the absence of a control group.

\section{Conclusions}

Contrary to expectations, we found no worsening in QoL and sleep before and after COVID-19 lockdown, as measured with standardized FMS QoL and sleep tools in this sample of FMS patients. Conversely, we noted an emotional impact, as measured with the IES-R, on a significant number of patients with psychological distress, which was not detected by commonly used QoL tests. These findings suggest the need to administer specific psychological tools in addition to standardized evaluation in FMS patient.

\section{AUTHOR CONTRIBUTIONS}

VS, ES and GP conceived of the presented idea, developed the theory and performed the computations. AM, KD, LG and GDB discussed the results and contributed to the final manuscript. GV and EP supervised the findings of this work. All authors read and approved the final manuscript.

\section{ETHICS APPROVAL AND CONSENT TO PARTICIPATE}

The study was approved by the local Clinical Research Committee (COVIDFIBRO study, ID 2890CESC) and complied with the revised ethical guidelines of the Declaration of Helsinki.

\section{ACKNOWLEDGMENT}

The Authors thank Dr. Maria Trainotti for her contribution to data collection and processing.

\section{FUNDING}

This research received no external funding.

\section{CONFLICT OF INTEREST}

The authors declare no conflict of interest. Giustino Varrassi is a member of the Editorial Board of this journal.

\section{DATA AVAILABILITY}

The data used to support the findings of this study are available from the corresponding author upon request.

\section{REFERENCES}

[1] Clauw DJ, Arnold LM, McCarberg BH. The Science of Fibromyalgia. Mayo Clinic Proceedings. 2011; 86: 907-911.

[2] Schweiger V, Del Balzo G, Raniero D, De Leo D, Martini A, Sarzi-Puttini $\mathrm{P}$, et al. Current trends in disability claims due to fibromyalgia syndrome. Clinical and Experimental Rheumatology. 2017; 35: 119-126.
[3] Coaccioli S, Varrassi G, Sabatini C, Marinangeli F, Giuliani M, Puxeddu A. Fibromyalgia: nosography and therapeutic perspectives. Pain Practice. 2008; 8: 190-201.

[4] Rahman A, Underwood M, Carnes D. Fibromyalgia. British Medical Journal. 2014; 348: g1224.

[5] Yasui M, Yoshimura T, Takeuchi S, Tokizane K, Tsuda M, Inoue K, et al. A chronic fatigue syndrome model demonstrates mechanical allodynia and muscular hyperalgesia via spinal microglial activation. Glia. 2015; 62: 1407-1417.

[6] Salaffi F, Atzeni F, Talotta R, Di Carlo M, Sarzi-Puttini P. Earthquake vulnerability of fibromyalgia patients: six-month follow-up after the catastrophic disasters in central Italy. Clinical and Experimental Rheumatology. 2017; 35: 93-99.

[7] Usui C, Hatta K, Aratani S, Yagishita N, Nishioka K, Okamura S, et al. Vulnerability to traumatic stress in fibromyalgia patients: 19 month follow-up after the great East Japan disaster. Arthritis Research \& Therapy. 2013; 15: R130.

[8] Allen SF, Stevenson J, Lazuras L, Akram U. The role of the COVID-19 pandemic in altered psychological well-being, mental health and sleep: an online cross-sectional study. Psychology, Health \& Medicine. 2021; $1-9$.

[9] Souza LFF, Paineiras-Domingos LL, Melo-Oliveira MES, PessanhaFreitas J, Moreira-Marconi E, Lacerda ACR, Mendonça VA, Sá-Caputo DDC, Bernardo-Filho M. The impact of COVID-19 pandemic in the quality of sleep by Pittsburgh Sleep Quality Index: a systematic review. Ciência \& Saúde Coletiva. 2021; 26: 1457-1466.

[10] Soares LL, Leite LB, Guilherme LQ, Rezende LM, Noce F, Pussieldi G. Anxiety, sleep quality and mood in elite athletes during the COVID19 pandemic: a preliminary study. The Journal of Sports Medicine and Physical Fitness. 2021. (in press)

[11] Sonza A, Da Cunha de Sá-Caputo D, Bachur JA, Rodrigues de Araújo MDG, Valadares Trippo KVT, Ribeiro Nogueira da Gama DRNDG, et al. Brazil before and during COVID-19 pandemic: impact on the practice and habits of physical exercise. Acta BioMedica. 2020; 92: e2021027.

[12] Melo-Oliveira ME, Sá-Caputo D, Bachur JA, Paineiras-Domingos LL, Sonza A, Lacerda AC, et al. Reported quality of life in countries with cases of COVID19: a systematic review. Expert Review of Respiratory Medicine. 2021; 15: 213-220.

[13] Bennett RM, Friend R, Jones KD, Ward R, Han BK, Ross RL. The Revised Fibromyalgia Impact Questionnaire (FIQR): validation and psychometric properties. Arthritis Research \& Therapy. 2009; 11: R120.

[14] Hoffman DL, Dukes EM. The health status burden of people with fibromyalgia: a review of studies that assessed health status with the SF36 or the SF-12. International Journal of Clinical Practice. 2008; 62: 115126.

[15] Osorio CD, Gallinaro AL, Lorenzi-Filho G, Lage LV. Sleep quality in patients with fibromyalgia using the Pittsburgh Sleep Quality Index. Journal of Rheumatology. 2006; 33: 1863-1865.

[16] Craparo G, Faraci P, Rotondo G, Gori A. The Impact of Event Scale Revised: psychometric properties of the Italian version in a sample of flood victims. Neuropsychiatric Disease and Treatment. 2013; 9: 14271432 .

[17] Martini A, Schweiger V, Del Balzo G, Sartori G, Parolini M, Chinellato $\mathrm{E}$, et al. Epidemiological, pharmacological and sociodemographic characterization of a population of 386 fibromyalgic patients referred to a tertiary pain centre in Italy. Clinical and Experimental Rheumatology. 2019; 37: 27-38.

[18] Osimo SA, Aiello M, Gentili C, Ionta S, Cecchetto C. The influence of personality, resilience, and alexithymia on mental health during COVID19 Pandemic. Frontiers in Psychology. 2021; 4: 630751.

[19] Cecchetto C, Aiello M, Gentili C, Ionta S, Osimo SA. Increased emotional eating during COVID-19 associated with lockdown, psychological and social distress. Appetite. 2021; 160: 105122.

[20] Conversano C, Marchi L, Rebecca C, Carmassi C, Contena B, Bazzichi LM, et al. Personality Traits in Fibromyalgia (FM): does FM personality exists? a Systematic Review. Clinical Practice and Epidemiology in Mental Health. 2018; 14: 223-232.

[21] Cavalli G, Cariddi A, Ferrari J, Suzzi B, Tomelleri A, Campochiaro $\mathrm{C}$, et al. Living with fibromyalgia during the COVID-19 pandemic: 
mixed effects of prolonged lockdown on the well-being of patients. Rheumatology. 2021; 60: 465-467.

[22] Jeppesen SS, Bentsen KK, Jørgensen TL, Holm HS, Holst-Christensen L, Tarpgaard LS, et al. Quality of life in patients with cancer during the COVID-19 pandemic - a Danish cross-sectional study (COPICADS). Acta Oncologica. 2021; 60: 4-12.

[23] Ciążynska M, Pabianek M, Szczepaniak K, Ułanska M, Skibinska M, Owczarek W, et al. Quality of life of cancer patients during coronavirus disease (COVID-19) pandemic. Psycho-Oncology. 2020; 1-3.

[24] Deledda G, Riccardi N, Gori S, Poli S, Giansante M, Geccherle E, et al. The impact of the SARS-CoV-2 outbreak on the psychological flexibility and behavior of cancelling medical appointments of italian patients with pre-existing medical condition: The "ImpACT-COVID-19 for patients" multi-centre observational study. International Journal of Environmental Research and Public Health. 2021; 18: 340.

[25] Passavanti M, Argentieri A, Barbieri DM, Lou B, Wijayaratna K, Foroutan Mirhosseini AS, et al. The psychological impact of COVID19 and restrictive measures in the world. Journal of Affective Disorders. 2021; 283: 36-51.

[26] Regier DA, Kuhl EA, Kupfer DJ. The DSM-5: Classification and criteria changes. World Psychiatry. 2013; 12: 92-98.

[27] O'Donnell ML, Agathos JA, Metcalf O, Gibson K, Lau W. Adjustment Disorder: Current Developments and Future Directions. International Journal of Environmental Research and Public Health. 2019; 16: 2537.
[28] Arnold LM, Crofford LJ, Mease PJ, Burgess SM, Palmer SC, Abetz L, et al. Patient perspectives on the impact of fibromyalgia. Patient Education and Counseling. 2008; 73: 114-120.

[29] Jenkinson C, Peto V, Fitzpatrick R, Greenhall R, Hyman N. Selfreported functioning and well-being in patients with Parkinson's disease: comparison of the short-form health survey (SF-36) and the Parkinson's Disease Questionnaire (PDQ-39) Age and Ageing. 1995; 24: 505-509.

[30] Spaeth M, Rizzi M, Sarzi-Puttini P. Fibromyalgia and sleep. Best Practice \& Research. Clinical Rheumatology. 2011; 25: 227-239.

[31] Hruschak V, Flowers KM, Azizoddin DR, Jamison RN, Edwards RR, Schreiber KL. Cross-sectional study of psychosocial and pain-related variables among patients with chronic pain during a time of social distancing imposed by the coronavirus disease 2019 pandemic. Pain. 2021; 162: 619-629.

How to cite this article: Vittorio Schweiger, Erica Secchettin, Giovanni Perini, Alvise Martini, Katia Donadello, Leonardo Gottin, et al. Quality of life and psychological assessment in patients with Fibromyalgia Syndrome during COVID-19 pandemic in Italy: prospective observational study. Signa Vitae. 2022;18(1):41-46. doi:10.22514/sv.2021.127. 